\title{
Particle-hole symmetric localization in optical lattices using time modulated random on-site potentials
}

\author{
Yue Zou (邹 悦), ${ }^{1}$ Ryan Barnett, ${ }^{2}$ and Gil Refael ${ }^{1}$ \\ ${ }^{1}$ Department of Physics, California Institute of Technology, Pasadena, California 91125, USA \\ ${ }^{2}$ Joint Quantum Institute and Condensed Matter Theory Center, Department of Physics, University of Maryland, \\ College Park, Maryland 20742, USA
}

(Received 22 July 2010; published 15 December 2010)

\begin{abstract}
The random hopping models exhibit many fascinating features, such as diverging localization length and density of states as energy approaches the band center due to its particle-hole symmetry. Nevertheless, such models are yet to be realized experimentally because the particle-hole symmetry is easily destroyed by diagonal disorder. Here we propose that a pure random hopping model can be effectively realized in ultracold atoms by modulating a disordered onsite potential in particular frequency ranges. This idea is motivated by the recent development of the phenomena called "dynamical localization" or "coherent destruction of tunneling." Investigating the application of this idea in one dimension, we find that if the oscillation frequency of the disorder potential is gradually increased from zero to infinity, one can tune a noninteracting system from an Anderson insulator to a random hopping model with diverging localization length at the band center, and eventually to a uniform-hopping tight-binding model.
\end{abstract}

DOI: 10.1103/PhysRevB.82.224205

PACS number(s): $03.75 . \mathrm{Hh}, 03.75 . \mathrm{Ss}, 71.55 . \mathrm{Jv}$

\section{INTRODUCTION}

The interplay of quenched disorder and low dimensionality in quantum systems has been a central theme in condensed-matter physics. A beautiful theory describes how the spectrum of disordered Hamiltonians follows from the symmetries of the problem. ${ }^{1}$ In this work, we focus on the one-dimensional noninteracting random hopping model, namely, the tight-binding model with pure off-diagonal disorder. This model's properties have been extensively investigated theoretically using various techniques for many years. This model also exhibits physics manifest in several other models, such as quantum particles connected by random strength springs, spin $1 / 2$ random $X X$ chains, random quantum Ising chains in a transverse field, and random mass Dirac fermions. This model exhibits many surprising features. Early theoretical work ${ }^{2-5}$ focused on properties derivable from the mean local Green's function, notably the typical localization length which diverges as $\sim \ln |E|$ and the mean density of states (DOS) also diverging as $\sim 1 /\left|E\left[\ln \left(E^{2}\right)\right]^{3}\right|$ as energy $E$ approaches the band center. Such behavior is very different from Anderson insulators where disorder appears in the diagonal terms of the Hamiltonian, and there are no singularities in the localization length or density of states spectrum. More recent work has studied this model using real-space renormalization group ${ }^{6}$ and supersymmetry methods ${ }^{7}$ and has uncovered more interesting results, most importantly, an additional length scalemean localization length as a function of energy-which diverges as $\sim \ln ^{2}|E|$. Most recently, the effect of random hopping amplitudes on interacting fermionic and bosonic systems has also been investigated. In the two-dimensional (2D) fermionic case,${ }^{8-10}$ it has been shown that random hopping amplitude, on top of nontrivial spectral effects, could lead to a type of instability; in the bosonic case, ${ }^{11,12}$ a "Mott glass" phase has been predicted in addition to usual Mott insulating and superfluid phases.
On the other hand, the experimental realization of a pure random hopping model has proven to be extremely difficult. This is mainly because diagonal disorder inevitably arises when we try to disorder bond values, and any amount of diagonal disorder would break the particle-hole symmetry of the random-hopping model and thereby destroy the universal behavior of this class of disordered Hamiltonians.

In this work, we propose that a pure random hopping model can be realized in optical lattices by first creating an Anderson insulator and then modulating the disordered onsite potential energy periodically. Our idea is closely connected to recent work on the phenomena dubbed "dynamical localization" or "coherent destruction of tunneling" in double wells, semiconductor superlattices, and recently optical lattices. ${ }^{13-33}$ While one can use this path to the random hopping model in any dimensionality $(d \leq 3)$, we will concentrate on its 1d application below.

The basic idea of dynamical localization is the following. Consider a single particle (either boson or fermion) in a double-well potential with a tunneling amplitude $J$ and a time-modulated potential-energy offset:

$$
H=-J\left(a^{\dagger} b+b^{\dagger} a\right)+\tilde{V} \cos (\omega t)\left(a^{\dagger} a-b^{\dagger} b\right) .
$$

By performing a unitary transformation with

$$
U=e^{-i(\tilde{V} / \omega) \sin (\omega t)\left(a^{\dagger} a-b^{\dagger} b\right)},
$$

one can readily obtain that in the large- $\omega$ limit, the original Schrödinger equation is transformed to the effective Hamiltonian

$$
H_{\text {eff }}=-J \mathcal{J}_{0}\left(\frac{\tilde{V}}{\omega}\right)\left(a^{\dagger} b+b^{\dagger} a\right),
$$

where $\mathcal{J}_{0}$ is the zeroth Bessel function. Thus one can see that the effect of time modulating the potential energy is to simply renormalize the tunneling amplitude $J$ in the large- $\omega$ 


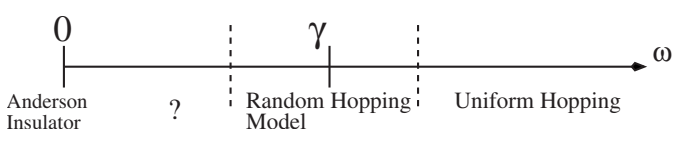

FIG. 1. Phase diagram of model (4) studied in this work. At zero frequency, the system is an Anderson insulator; when the frequency $\omega$ is comparable to the disorder width $\gamma$, the system behaves as a random hopping model; when $\omega$ is much larger than $\gamma$, the system crosses over to the uniform-hopping tight-binding regime, which is fully achieved when $\omega=\infty$. We also present some interesting and puzzling results for the regime $0<\omega \ll \gamma$.

limit. The frequency $\omega$ can be fine tuned such that $\mathcal{J}_{0}\left(\frac{\widetilde{V}}{\omega}\right)$ $=0$, and the system becomes localized if there is also an interaction term. This is the so-called dynamical localization phenomenon, and it has been observed in experiments. ${ }^{27,28,33}$ It has also been proposed as a method to tune interacting bosons through the superfluid-insulator transition, ${ }^{21,23}$ to observe the analog of photon assisted tunneling and Shapiro steps, ${ }^{16,22}$ and to manipulate the localization properties of Anderson insulators. ${ }^{17,24}$ Recent experimental work has confirmed some of these proposals. ${ }^{34,35}$

In this work, we will use the term dynamical localization to denote the type of mathematical transformations that takes Hamiltonian (2) to effective Hamiltonian (3), rather than a specific localization phenomenon. For our purpose, it suffices to notice that the original potential energy $\tilde{V}$ resides in the renormalization factor of the hopping amplitude. Thus, if one modulates an Anderson insulator instead, one expects that the disorder in the onsite energy should be transformed into the disorder of hopping amplitude in the same way. In other words, one obtains the random hopping model by rapidly modulating the disordered potential energies of an Anderson insulator. As we will see in later sections, however, if the modulating frequency $\omega$ is much larger than typical potential energy, this randomness in effective hopping amplitude is suppressed, and we obtain a uniform-hopping tightbinding model. Therefore, the random hopping model behavior survives when the frequency $\omega$ is comparable to the typical potential energy. In summary, as the oscillation frequency of the potential energy is gradually increased from zero to infinity, one can tune a noninteracting system from an Anderson insulator to a random hopping model with diverging localization length at the band center, and eventually to a uniform-hopping tight-binding model (see Fig. 1).

Note, also, that a different but related model was studied in Refs. 17, 24, and 25, where an Anderson insulator with stationary disordered potentials but oscillating linear potential (e.g., uniform ac electric field) is considered. In that model, the localization properties of the Anderson insulator can be manipulated by the oscillating linear potential, but the random hopping model behavior is not accessible.

In the remainder of this work, we will analyze the localization properties of the following model:

$$
\begin{gathered}
H=H_{0}+2 V \cos (\omega t), \\
H_{0}=-J \sum_{n=1}^{N-1}\left(c_{n}^{\dagger} c_{n+1}+c_{n+1}^{\dagger} c_{n}\right),
\end{gathered}
$$

$$
V=\sum_{n=1}^{N} v_{n} c_{n}^{\dagger} c_{n}
$$

where $N$ is the system size, and we assumed the onsite potential $v_{n}$ obeys a uniform distribution between $[-\gamma / 2, \gamma / 2]$. We will work in single particle case and neglect interactions, and thus the statistics of the particles does not matter. In Sec. II, we will introduce the Floquet formalism with which we obtain the localization length and the density of states of the time-dependent Hamiltonian (4). In Sec. III, an effective time-independent Hamiltonian is shown to emerge from the high-frequency limit of the original time-dependent Hamiltonian (4) in analogy to the dynamical localization phenomena. Numerical results from both the Floquet calculation and the effective Hamiltonian calculation are presented and discussed in detail in Sec. IV. Next, we discuss possible experimental methods to modulate on-site disorder and to detect signatures of random hopping models in optical lattices. Finally, we summarize our results in Sec. VI.

\section{COMPUTATION OF THE DENSITY OF STATES AND THE LOCALIZATION LENGTH}

For a time-periodic system with Hamiltonian $H(t)$ and period $T=2 \pi / \omega$, by the Floquet theorem, its wave functions can be written in the form

$$
\psi(t)=e^{-i E t} \phi(t)
$$

where $E$ is the quasienergy defined modulo $\omega$ and $\phi(t+T)$ $=\phi(t)$. Here and in what follows we have set $\hbar=1$. This well-known result is the analog of the Bloch's theorem for particles in a periodic spatial potential. To solve for $E$ and $\phi(t)$, one approach is to rewrite the Schrodinger equation

$$
i \partial_{t} \psi(t)=H \psi(t)
$$

as

$$
H_{F} \phi=E \phi,
$$

where $H_{F}$ is the so-called Floquet Hamiltonian

$$
H_{F}=H-i \partial t \text {, }
$$

which is a matrix in the augmented Hilbert space $\mathcal{H} \times \mathcal{T}$, where $\mathcal{H}$ is the original Hilbert space and $\mathcal{T}$ is the frequency space, ${ }^{36,37}$ and then to find the eigenvalues and eigenstates of $H_{F}$. Alternatively, it is also well known that $e^{-i E T}$ and $\phi(T)$ are the eigenvalue and eigenstate of the Floquet operator

$$
\mathcal{F}=\tilde{\mathcal{T}} \exp \left(-i \int_{0}^{T} d t H(t)\right),
$$

where $\tilde{\mathcal{T}}$ is the time-ordering operator.

To obtain the density of states, we work with the latter approach. First, we calculate the Floquet operator $\mathcal{F}$ by the numerical Trotterization procedure. Then, we diagonalize the Floquet operator $\mathcal{F}$ to find the quasienergies which we define to be in the "first Brillouin zone" $-\omega / 2 \leq E \leq \omega / 2$. Then, we obtain the cumulative distribution function of the quasienergies, average it over many realization of disorder, numeri- 
cally differentiate it with respect to quasienergy, and finally obtain the density of states.

We would also like to obtain the localization length of this model for arbitrary frequency $\omega$. For one-dimensional noninteracting time-independent systems with $N$ sites, we recall that the localization length of a state with energy $E$ is given $\mathrm{by}^{3}$

$$
\frac{1}{\lambda(E)}=-\lim _{N \rightarrow \infty} \frac{1}{N} \ln \left|G_{1 N}(E)\right|,
$$

where the Green's function

$$
G(E)=(E I-H)^{-1},
$$

$I$ is the identity matrix.

Following Refs. 25 and 38, we generalize the concept of localization length of a time-periodic system by defining it as the localization length of the time-averaged wave function. In terms of the Green's function, it is

$$
\frac{1}{\lambda(E)}=-\lim _{N \rightarrow \infty} \frac{1}{N} \ln \left|\tilde{G}_{1 N}(E)\right|,
$$

where

$$
\widetilde{G}=\left\langle\Omega=0\left|G_{F}(E)\right| \Omega=0\right\rangle
$$

and $G_{F}$ is the so-called Floquet Green's function:

$$
G_{F}(E)=\left(E I-H_{F}\right)^{-1} .
$$

Here, if we denote the frequency operator $\hat{\Omega}=i \partial_{t}$ and its eigenstates

$$
\hat{\Omega}|n\rangle=n \omega|n\rangle,
$$

$|\Omega=0\rangle$ introduced above is simply the eigenstate with $n=0$.

Next, we discuss how to compute $\widetilde{G} \equiv\left\langle\Omega=0\left|G_{F}(E)\right| \Omega\right.$ $=0\rangle$. For a Hamiltonian of the form

$$
H=H_{0}+2 V \cos (\omega t)
$$

from

$$
\left(E I-H_{F}\right) G_{F}=I,
$$

we insert the resolution of identity in the frequency space and obtain

$$
\sum_{p}\left\langle m\left|\left(E I-H_{F}\right)\right| p\right\rangle\left\langle p\left|G_{F}\right| n\right\rangle=I\langle m \mid n\rangle .
$$

Thus,

$$
\left[(E+m \omega) I-H_{0}\right] G_{m n}-V G_{m+1, n}-V G_{m-1, n}=I \delta_{m n},
$$

where

$$
G_{m n} \equiv\left\langle m\left|G_{F}\right| n\right\rangle .
$$

To solve for $\widetilde{G}(E)$ which is $G_{00}(E)$ from this system of equations, we follow Ref. 25 to obtain

$$
\widetilde{G}(E) \equiv G_{00}(E)=\left(E I-H_{0}-V_{\text {eff }}^{+}-V_{\text {eff }}^{-}\right)^{-1},
$$

where

$$
V_{\mathrm{eff}}^{ \pm}=V \frac{1}{E \pm 1 \omega-H_{0}-V \frac{1}{E \pm 2 \omega-H_{0}-V \frac{1}{\vdots} V} V} V,
$$

or equivalently,

$$
V_{\mathrm{eff}}^{ \pm}=V \frac{1}{E \pm 1 \omega-H_{0}-V_{1}^{ \pm}} V,
$$

where for $n=1,2,3, \ldots$,

$$
V_{n}^{ \pm}=V \frac{1}{E \pm(n+1) \omega-H_{0}-V_{n+1}^{ \pm}} V .
$$

The number of iterations needed to ensure the convergence of $V_{\mathrm{eff}}^{ \pm}$is roughly proportional to $\gamma / \omega$.

Finally, it would be interesting to mathematically check if the Thouless relation ${ }^{3}$ between the density of states and the localization length holds for a Floquet system.

\section{EFFECTIVE HAMILTONIAN IN THE FAST OSCILLATION LIMIT}

In this section, we show that if the oscillation frequency $\omega$ is comparable or larger than the disorder width $\gamma$, the original Schrodinger equation of a time-dependent Hamiltonian can be transformed to that of a time-independent effective Hamiltonian. For the original Schrodinger equation

$$
i \partial_{t} \psi=H \psi, \quad H=H_{0}+2 V \cos (\omega t),
$$

we define

$$
\psi=U \tilde{\psi}, \quad U=e^{-2 i \sin (\omega t) V / \omega} .
$$

Then the Schrodinger equation becomes

$$
i \partial_{t} \tilde{\psi}=H_{\mathrm{eff}} \tilde{\psi}, \quad H_{\mathrm{eff}}=U^{\dagger} H U-2 V \cos (\omega t) .
$$

Using the operator identity (valid for both bosons and fermions)

$$
e^{i \eta c_{n}^{\dagger} c_{n}} c_{n} e^{-i \eta c_{n}^{\dagger} c_{n}}=c_{n} e^{-i \eta}
$$

we have

$$
\begin{aligned}
H_{\mathrm{eff}}= & -J \sum_{n}\left[\left(c_{n}^{\dagger} c_{n+1}+c_{n+1}^{\dagger} c_{n}\right) \sum_{m=-\infty}^{\infty}(-1)^{m}\right. \\
& \times \mathcal{J}_{m}\left(\frac{2\left(v_{n}-v_{n+1}\right)}{\omega}\right) \cos (m \omega t)+i\left(c_{n}^{\dagger} c_{n+1}-c_{n+1}^{\dagger} c_{n}\right) \\
& \left.\times \sum_{m=-\infty}^{\infty}(-1)^{m+1} \mathcal{J}_{m}\left(\frac{2\left(v_{n}-v_{n+1}\right)}{\omega}\right) \sin (m \omega t)\right] .
\end{aligned}
$$

For $\omega$ larger than or comparable to $\gamma$, the argument of the Bessel functions is comparable to or smaller than 1. Hence $\mathcal{J}_{0}$ dominates over other Bessel functions, and we obtain an effective time-independent Hamiltonian 


$$
H_{\mathrm{eff}}^{(0)} \approx-J \sum_{n}\left(c_{n}^{\dagger} c_{n+1}+c_{n+1}^{\dagger} c_{n}\right) \mathcal{J}_{0}\left(\frac{2\left(v_{n}-v_{n+1}\right)}{\omega}\right),
$$

which is a tight-binding model with a site-dependent effective hopping amplitude

$$
J_{\text {eff }, n}=J \mathcal{J}_{0}\left(\frac{2\left(v_{n}-v_{n+1}\right)}{\omega}\right) .
$$

When $\omega$ is not too large, this is exactly the random hopping model we are looking for, and it should exhibit behaviors such as diverging localization length and density of states at the band center. We can compute the localization length of this effective Hamiltonian by using Eq. (10) and compare with the exact calculation using Eq. (10):

$$
\begin{gathered}
\frac{1}{\lambda(E)}=-\lim _{N \rightarrow \infty} \frac{1}{N} \ln \left|G_{1 N}(E)\right|, \\
G_{\mathrm{eff}}(E)=\left(E I-H_{\mathrm{eff}}^{(0)}\right)^{-1} .
\end{gathered}
$$

It is also straightforward to compute the density of states of this model numerically.

However, when $\omega \gg \gamma$,

$$
\mathcal{J}_{0}\left(\frac{2\left(v_{n}-v_{n+1}\right)}{\omega}\right) \approx 1
$$

regardless of the value of $v_{n}$. Therefore in this limit the system behaves like a uniform-hopping tight-binding model (see Fig. 1). In this regime, we expect the localization length of every state to diverge, and the density of states diverges at the band edges instead.

Finally, as long as the effective Hamiltonian (27) is a good approximation of the original Hamiltonian, we can see from its form that physical properties are functions of $\left(v_{n}\right.$ $\left.-v_{n+1}\right) / \omega$, which in turn are functions of the ratio $\gamma / \omega$ (recall that $v_{n}$ is a random variable with uniform distribution in [ $-\gamma / 2, \gamma / 2])$.

\section{NUMERICAL RESULTS}

We have computed the localization length and the density of states for various values of the frequency $\omega$ with fixed hopping amplitude $J=1$ and disorder width $\gamma=10$. The results from the Floquet technique for the original Hamiltonian (4) and those obtained from the effective Hamiltonian (27) at $\omega=3,7,35$ are plotted in Fig. 2. One can see that when $\omega$ $=7$ which is comparable to the disorder width $\gamma$ and when $\omega=35$ which is much larger than $\gamma$, the results from the exact Floquet calculation and those from effective Hamiltonian calculation agree quite well. At $\omega=35 \gg \gamma=10$, every state is completely delocalized, and the density of states diverges at the two band edges, as expected for a uniform-hopping tightbinding model. On the other hand, at $\omega=7$, both the localization length and the density of states diverge at the band center, which are characteristic of the random hopping model, as expected. The case of $\omega=3$ is slightly more surprising: although the effective Hamiltonian does not work well, the system still exhibits diverging localization length
Inverse Localization Length Inverse Density Of States



(a)

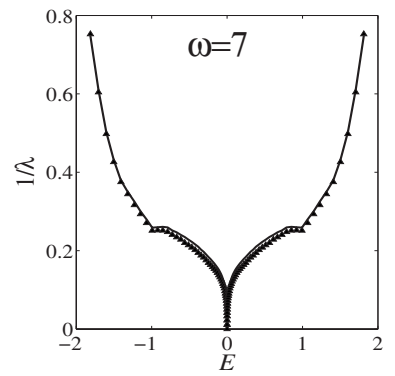

(c)

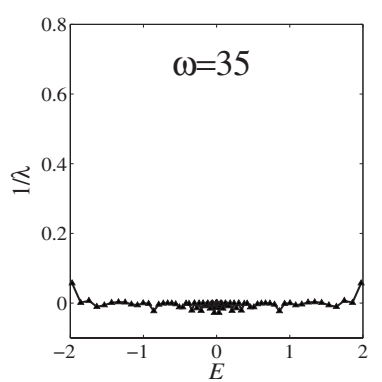

(e)



(b)

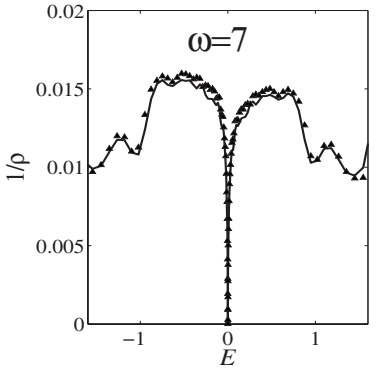

(d)



(f)
Inverse Localization Length Inverse Density Of States

FIG. 2. [(a), (c), and (e)] Inverse of the localization length $1 / \lambda$ and $[(\mathrm{b}),(\mathrm{d})$, and (f)] inverse of the density of states $1 / \rho$ vs quasienergy $E$ (in units of the hopping amplitude $J$ ). Disorder width $\gamma$ $=10 J$, hopping amplitude $J=1$, oscillation frequency $\omega$ $=3 J, 7 J, 35 J$. Solid lines are from the Floquet calculation of the original model (4); solid triangles are from the effective Hamiltonian $H_{\mathrm{eff}}^{(0)}(27)$. Averaged over 1000 realizations of disorder.

and density of states at the band center. In Fig. 3, we plot the localization length for more values of $\omega$ from 3 to 35 , and the trend from random hopping model behavior to uniformhopping tight-binding model as $\omega$ increases is clearly seen.

Near the band center, we fit the results of localization and the density of states to well-known analytical results ${ }^{5}$ (see Fig. 4)

$$
\begin{gathered}
\rho(E)=N \cdot \frac{2 \sigma^{2}}{\left|E\left\{\ln \left[\left(E / E_{0}\right)^{2}\right]\right\}^{3}\right|}, \\
\lambda(E)=\frac{2\left|\ln \left[\left(E / E_{0}^{\prime}\right)^{2}\right]\right|}{\sigma^{2}},
\end{gathered}
$$

where $N$ is the system size, $E_{0}$ and $E_{0}^{\prime}$ are energy scales which we treat as fitting parameters, and $\sigma$ is the standard 


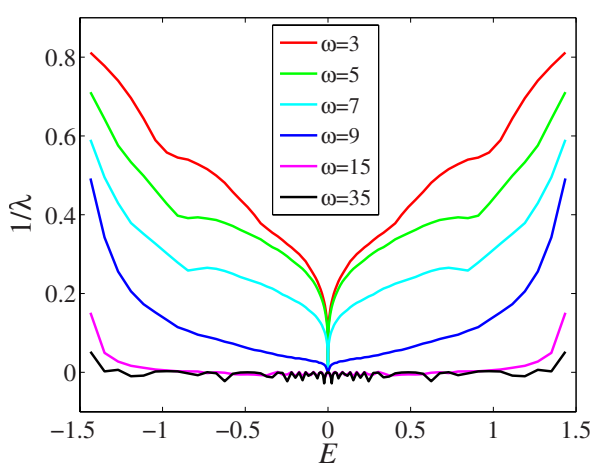

FIG. 3. (Color online) Inverse of the localization length $1 / \lambda$ vs quasienergy $E$ (in units of the hopping $J$ ). Averaged over 1000 realizations of disorder. Hopping amplitude $J=1$, disorder width $\gamma$ $=10 \mathrm{~J}$, oscillation frequency $\omega=3 \mathrm{~J}, 5 \mathrm{~J}, 7 \mathrm{~J}, 9 \mathrm{~J}, 15 \mathrm{~J}, 35 \mathrm{~J}$.

deviation of the logarithm of the effective hopping amplitude square $\ln J_{\text {eff }}^{2}$, with [see Eq. (28)]

$$
J_{\mathrm{eff}, n}=J \mathcal{J}_{0}\left[\frac{2\left(v_{n}-v_{n+1}\right)}{\omega}\right] .
$$

We can easily evaluate $\sigma$ numerically to be 1.535 given $\omega$ $=7$ and $\gamma=10$. Fitting numerical results of localization length and density of states, we obtain
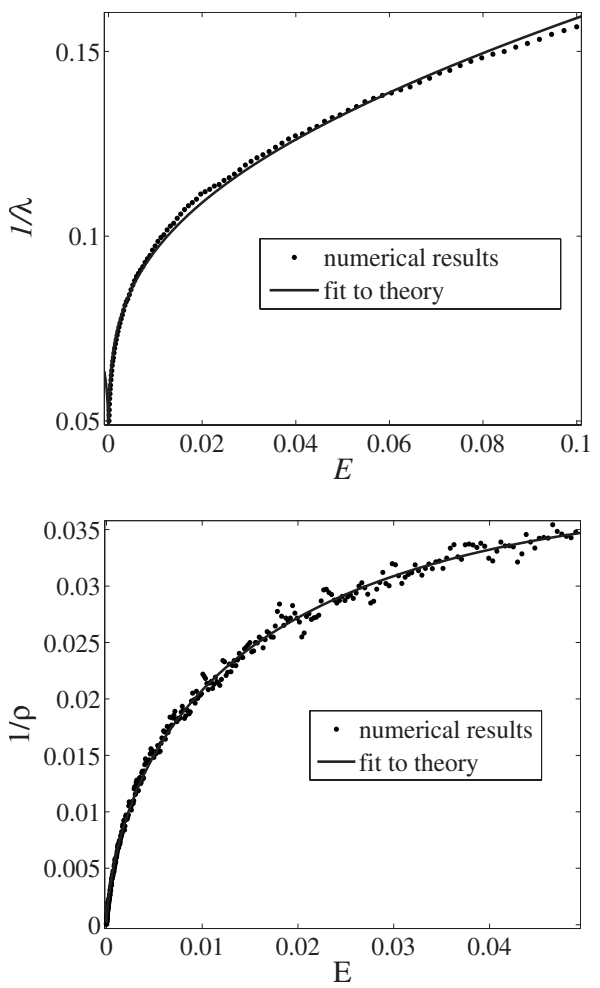

FIG. 4. Fitting the inverse of the localization length $\lambda^{-1}$ and the inverse of the density of states $\rho^{-1}$ near the band center to their analytical form $\rho(E)=N 2 \sigma^{2} /\left|E\left[\ln \left(E / E_{0}\right)^{2}\right]^{3}\right|$ and $\lambda(E)$ $=2\left|\ln \left(E / E_{0}^{\prime}\right)^{2}\right| / \sigma^{2}$. Here, $E_{0}$ and $E_{0}^{\prime}$ are energy scales (Ref. 5), and the theoretical value of $\sigma$ is the standard deviation of $\ln J_{\text {eff }}^{2}$, which is 1.535 for our choice of parameters here. The fitted value of $\sigma$ is 1.496 for $1 / \lambda$ and 1.677 for $1 / \rho$. Hopping amplitude $J=1$, oscillation frequency $\omega=7$, and disorder width $\gamma=10$.

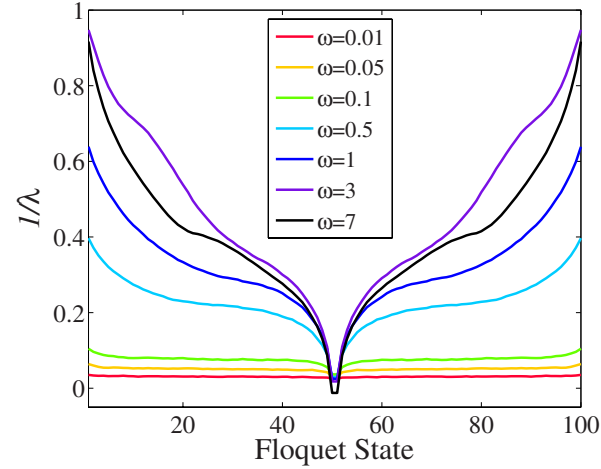

FIG. 5. (Color online) Inverse of the localization length $1 / \lambda$ vs the label (e.g., 1st, 2nd, 3rd, ..., 100th) of every Floquet eigenstate for $\omega=0.05,0.1,0.5,1,3,7$. System size $N=100$, hopping amplitude $J=1$, oscillation frequency $\omega=7$, and disorder width $\gamma=10$.

$$
\sigma_{\text {fit }, \lambda}=1.496, \quad \sigma_{\text {fit }, \rho}=1.677,
$$

which are quite close to the theoretical value 1.535 obtained above, further confirming our expectation that random hopping model behavior can be achieved by fast modulating the onsite energy of Anderson insulators.

At frequencies much smaller than $\gamma$ and the original hopping strength $J$, interestingly, the system has quite large localization length. In Fig. 5, we plot the inverse of the localization length vs the label (e.g., 1st, 2nd, 3rd, ..., 100th) of every Floquet eigenstate for $\omega=0.01,0.05,0.1,0.5,1,3,7$ with system size $N=100$ (the total number of Floquet states equals the system size $N$ ). One can see that from $\omega=7$, when $\omega$ is lowered, first the localization length decreases (inverse of the localization increases), but around $\omega=3$ this trend is reversed, and all the states become more and more delocalized at smaller frequencies. At $\omega=0.01,0.05$, and 0.1 , all the states have almost equally large localization length.

This trend of delocalization at small frequencies is quite puzzling, but it is interesting to notice that in a similar model where an Anderson insulator is manipulated with an ac electric field, ${ }^{17,24,25}$ an analogous delocalization trend was found. An intuitive argument was given in Ref. 25, where it is argued that the modulation with frequency $\omega$ allows electrons to absorb or emit integer numbers of "photons" with energy $\omega$. When $\omega$ is smaller, more states with quasienergy $E \pm n \omega$ are in the original energy band. Since the scale of the localization length of the new state should be set by the state with the largest localization length among all the states with energy $E \pm n \omega$, a smaller $\omega$ implies that it is more likely for the new state to have larger localization length. This intuitive picture could be of some relevance to our case here as well.

\section{EXPERIMENTAL FEASIBILITY}

The main motivation for our work is to experimentally realize the particle-hole symmetric disorder classes. Following the route outlined above, requires creating an Anderson insulator in optical lattices, and then modulate the onsite disorder potential periodically in time. The behavior of ultracold atomic systems subject to disorder, and the resulting localization phenomena is a field of intense current 
research. ${ }^{39}$ Experiments have relied on two methods to introduce disorder into such systems. The first involves using two incommensurate optical lattice potentials, providing an effective realization of the Aubrey Andre model which has been shown to give localization. ${ }^{40}$ The second method uses a speckle potential produced by passing a laser through a diffusing plate which directly imprints a disorder potential. ${ }^{41}$

The main challenge in realizing the phenomena introduced in this work is producing time-dependent disorder potentials which periodically attract and repel the atoms in the optical lattice system. The most direct way to achieve this is to periodically change the detuning of the disorder potential from red to blue. That is, the disorder potential is given by

$$
V(r)=\frac{3 \pi c^{2}}{2 \omega_{0}^{2}}\left(\frac{\Gamma}{\Delta(t)}\right) I(r),
$$

where $c$ is the speed of light, $\omega_{0}$ is the atomic resonance frequency, and $\Delta(t)=\omega(t)-\omega_{0}$ is the detuning frequency. Thus, the sign of the disorder potential can be periodically changed by periodically changing the detuning. This can be achieved by using an acousto-optic modulator to continuously vary the laser frequency. However, sweeping through the resonance can produce undesirable atomic losses. Thus it might be best to periodically alternate two laser beams (one red and the other blue detuned) through the same speckle potential.

The main experimental probe to detect Anderson localization in cold atom system has been time-of-flight spectroscopy. ${ }^{40,41}$ In particular, for weak disorder when the condensate occupies a delocalized state, the condensate ballistically expands when the confining potential is removed. On the other hand for strong disorder potentials, the condensate occupies localized states and ceases to expand at a characteristic time after released from the trap. As we have seen, in the random hopping model some of the states are localized while others are delocalized (near the band center). Thus disentangling such behavior using time-of-flight spectroscopy alone might prove to be an experimental challenge. On the other hand, the well-developed technique of Bragg spectroscopy ${ }^{42,43}$ allows the access to the spectral function and therefore the density of states of quantum gas. Thus, perhaps the most promising way of detecting the Dyson delocalized state is through its distinct single single-particle density of states near the band center given by Eq. (31) measured through Bragg spectroscopy.

\section{SUMMARY AND DISCUSSION}

The simple particle-hole symmetric class of quantum disorder problems exhibits many fascinating properties such as diverging localization length and diverging density of states at the band center. It is difficult, however, to realize it experimentally because its crucial particle-hole symmetry is destroyed by any amount of diagonal disorder. Our work suggests a realistic path to effectively realizing random hop- ping models in optical lattices by fast modulating the disordered potential energies of an Anderson insulator, but without need for fine tuning the local potential. Our idea follows naturally from the recently studied, so-called dynamical localization or coherent destruction of tunneling phenomena as well as from recent efforts to observe Anderson insulators in optical lattices by using various ways to artificially produce disordered potentials.

The setup we suggest can explore more than just the static properties of a special disorder model. After all, we are describing the response of a quantum system to a strong timedependent potential. By exact diagonalization of the Floquet operators arising from our model, we explored the spectral properties of a 1D system subject to strong time-periodic disorder. As shown in Figs. 2 and 3, the special features anticipated from the $1 \mathrm{D}$ random hopping problem arise in a wide range of frequency modulations. Even with moderately small frequency $\omega$ where the effective Hamiltonian (averaged in the vector-potential-only gauge) does not provide a satisfactory description, the localization length and the density of states still show the random-hopping-model behavior (see the case of $\omega=3$ in Fig. 2). Furthermore, a fit of our results near the band center to the well-known theoretical form $^{5}$ gives good agreement (see Fig. 4).

Our model, however, gives results which we do not yet intuitively understand in the low frequency limit. As one can see from Fig. 3, the localization length gradually decreases if the frequency $\omega$ is reduced from 35 to 3 given the disorder width $\gamma=10$. When the frequency $\omega$ is further lowered, our numerical results reveal that the localization length starts to increase again, and it becomes quite large for $\omega=0.05$ and $\omega=0.1$ (see Fig. 5). We should recall that the terminus of the limit $\omega \rightarrow 0$ is the well-known Anderson insulator, in which all states are localized. We intend to study the localization properties of such Floquet operators with strong disorder in future work.

While here we concentrated on the realization of particular classes of quantum disorder problems, the use of time modulated Hamiltonians could be a general path to the experimental realization of desirable systems. Indeed, this philosophy is already apparent in the quest to realize low-filling quantum Hall states in cold atom systems, ${ }^{44}$ and we expect that in both cold-atom and solid-state based devices, timedependent Hamiltonians will become a standard tool for the stabilization of unique many-body wave functions.

\section{ACKNOWLEDGMENTS}

For particularly useful discussions we thank J. Biddle, L. Fallani, S. Rolston, I. Spielman, and B. Wu. We would like to acknowledge support from the Joint Quantum Institute Physics Frontier Center and the Sherman Fairchild Foundation (R.B.); and the Packard Foundation, Sloan Foundation, and the research corporation, as well as Darpa, and NSF Grants No. PHY-0456720 and No. PHY-0803371 (G.R.). 
${ }^{1}$ M. R. Zirnbauer, J. Math. Phys. 37, 4986 (1996).

${ }^{2}$ F. J. Dyson, Phys. Rev. 92, 1331 (1953).

${ }^{3}$ D. J. Thouless, J. Phys. C 5, 77 (1972).

${ }^{4}$ G. Theodorou and M. H. Cohen, Phys. Rev. B 13, 4597 (1976).

${ }^{5}$ T. P. Eggarter and R. Riedinger, Phys. Rev. B 18, 569 (1978).

${ }^{6}$ D. S. Fisher, Phys. Rev. B 50, 3799 (1994).

${ }^{7}$ L. Balents and M. P. A. Fisher, Phys. Rev. B 56, 12970 (1997).

${ }^{8}$ R. Gade, Nucl. Phys. B 398, 499 (1993).

${ }^{9}$ O. Motrunich, K. Damle, and D. A. Huse, Phys. Rev. B 65 , 064206 (2002).

${ }^{10}$ M. S. Foster and A. W. W. Ludwig, Phys. Rev. B 77, 165108 (2008).

${ }^{11}$ E. Altman, Y. Kafri, A. Polkovnikov, and G. Refael, Phys. Rev. Lett. 93, 150402 (2004).

${ }^{12}$ E. Altman, Y. Kafri, A. Polkovnikov, and G. Refael, Phys. Rev. Lett. 100, 170402 (2008).

${ }^{13}$ D. H. Dunlap and V. M. Kenkre, Phys. Rev. B 34, 3625 (1986).

${ }^{14}$ F. Grossmann, T. Dittrich, P. Jung, and P. Hänggi, Phys. Rev. Lett. 67, 516 (1991)

${ }^{15}$ M. Holthaus, Phys. Rev. Lett. 69, 351 (1992).

${ }^{16}$ A. A. Ignatov, K. F. Renk, and E. P. Dodin, Phys. Rev. Lett. 70, 1996 (1993).

${ }^{17}$ M. Holthaus, G. H. Ristow, and D. W. Hone, Phys. Rev. Lett. 75, 3914 (1995).

${ }^{18}$ T. Meier, G. von Plessen, P. Thomas, and S. W. Koch, Phys. Rev. B 51, 14490 (1995).

${ }^{19}$ M. Grifoni and P. Hanggi, Phys. Rep. 304, 229 (1998).

${ }^{20}$ M. Holthaus, Phys. Rev. A 64, 011601 (2001).

${ }^{21}$ A. Eckardt, C. Weiss, and M. Holthaus, Phys. Rev. Lett. 95, 260404 (2005).

${ }^{22}$ A. Eckardt, T. Jinasundera, C. Weiss, and M. Holthaus, Phys. Rev. Lett. 95, 200401 (2005).

${ }^{23}$ C. E. Creffield and T. S. Monteiro, Phys. Rev. Lett. 96, 210403 (2006).

${ }^{24}$ D. F. Martinez and R. A. Molina, Phys. Rev. B 73, 073104 (2006).

${ }^{25}$ D. F. Martinez and R. A. Molina, Eur. Phys. J. B 52, 281 (2006).
${ }^{26}$ A. Eckardt and M. Holthaus, EPL 80, 50004 (2007).

${ }^{27}$ H. Lignier, C. Sias, D. Ciampini, Y. Singh, A. Zenesini, O. Morsch, and E. Arimondo, Phys. Rev. Lett. 99, 220403 (2007).

${ }^{28}$ E. Kierig, U. Schnorrberger, A. Schietinger, J. Tomkovic, and M. K. Oberthaler, Phys. Rev. Lett. 100, 190405 (2008).

${ }^{29}$ X. Luo, Q. Xie, and B. Wu, Phys. Rev. A 77, 053601 (2008).

${ }^{30}$ N. Tsukada, H. Yoshida, and T. Suzuki, Phys. Rev. A 77, 022101 (2008).

${ }^{31}$ Y. Kayanuma and K. Saito, Phys. Rev. A 77, 010101 (2008).

${ }^{32}$ C. E. Creffield, Phys. Rev. A 79, 063612 (2009).

${ }^{33}$ A. Eckardt, M. Holthaus, H. Lignier, A. Zenesini, D. Ciampini, O. Morsch, and E. Arimondo, Phys. Rev. A 79, 013611 (2009).

${ }^{34}$ C. Sias, H. Lignier, Y. P. Singh, A. Zenesini, D. Ciampini, O. Morsch, and E. Arimondo, Phys. Rev. Lett. 100, 040404 (2008).

${ }^{35}$ A. Zenesini, H. Lignier, D. Ciampini, O. Morsch, and E. Arimondo, Phys. Rev. Lett. 102, 100403 (2009).

${ }^{36}$ J. H. Shirley, Phys. Rev. 138, B979 (1965).

${ }^{37}$ H. Sambe, Phys. Rev. A 7, 2203 (1973).

${ }^{38}$ D. F. Martinez, J. Phys. A 36, 9827 (2003).

${ }^{39}$ L. Fallani, C. Fort, and M. Inguscio, arXiv:0804.2888 (unpublished).

${ }^{40}$ G. Roati, C. D'Errico, L. Fallani, M. Fattori, C. Fort, M. Zaccanti, G. Modugno, M. Modugno, and M. Inguscio, Nature (London) 453, 895 (2008).

${ }^{41}$ J. Billy, V. Josse, Z. Zuo, A. Bernard, B. Hambrecht, P. Lugan, D. Clment, L. Sanchez-Palencia, P. Bouyer, and A. Aspect, Nature (London) 453, 891 (2008).

${ }^{42}$ J. Stenger, S. Inouye, A. P. Chikkatur, D. M. Stamper-Kurn, D. E. Pritchard, and W. Ketterle, Phys. Rev. Lett. 82, 4569 (1999).

${ }^{43}$ D. M. Stamper-Kurn, A. P. Chikkatur, A. Görlitz, S. Inouye, S. Gupta, D. E. Pritchard, and W. Ketterle, Phys. Rev. Lett. 83, 2876 (1999).

${ }^{44}$ A. S. Sørensen, E. Demler, and M. D. Lukin, Phys. Rev. Lett. 94, 086803 (2005). 